\title{
Patients' and emergency clinicians' perceptions of improving prehospital pain management: a qualitative study
}

Mohammad Iqbal ${ }^{1}$, Anne Spaight ${ }^{1}$, and *Aloysius Niroshan Siriwardena ${ }^{1,2}$

Mohammad Iqbal MBBS, MCommH

Research Associate, East Midlands Ambulance Service NHS Trust

Lincolnshire Divisional Headquarters, Cross O'Cliff Court

Bracebridge Heath, Lincoln LN4 2HL, UK

Anne Spaight MA

Head of Clinical Governance, Audit and Research, East Midlands Ambulance Service

NHS Trust, UK

*Aloysius Niroshan Siriwardena MBBS MMedSci FRCGP PhD

Professor of Primary and Prehospital Health Care, University of Lincoln, UK

Associate Clinical Director, East Midlands Ambulance Service NHS Trust

Affiliated institutions:

${ }^{1}$ East Midlands Ambulance Service NHS Trust, UK

${ }^{2}$ University of Lincoln, Lincoln, UK

Keywords: pain, analgesia, prehospital care, paramedic, Emergency Medical Services

Running title: Qualitative study of prehospital pain management

Word count (excluding quotes and references): 2889

*Author for correspondence

A Niroshan Siriwardena

Professor of Primary and Prehospital Health Care

Faculty of Health, Life \& Social Sciences

University of Lincoln

Brayford Pool Lincoln LN6 7TS

Telephone: +44 (0) 1522886939

Fax: +44 (0) 1522837058

Email: nsiriwardena@lincoln.ac.uk 


\section{Patients' and emergency clinicians' perceptions of improving prehospital pain management: a qualitative study}

\section{ABSTRACT}

\section{Background}

We aimed to investigate patients' and practitioners' views and experiences of prehospital pain management to inform improvements in care and a patient centred approach to treatment.

\section{Methods}

This was a qualitative study involving a single emergency medical system. Data were gathered through focus groups and semi-structured interviews. Participants were purposively sampled from patients transported by ambulance to hospital with a painful condition during past six months, ambulance service and emergency department (ED) clinicians. Interviews were audiotaped, transcribed and thematic analysis was conducted.

\section{Results}

We interviewed 55 participants: 17 patients, 25 ambulance clinicians and 13 ED clinicians. Key themes included: (a) consider beliefs of patients and staff in pain management (b) widen pain assessment strategies (c) optimise non-drug treatment (d) increase drug treatment options and (e) enhance communication and coordination along the prehospital pain management pathway. Patients and staff expected pain to be relieved in the ambulance; however, refusal of or inadequate analgesia were common. Pain was commonly assessed using a verbal score but practitioners' views of severity were sometimes discordant with this. Morphine and Entonox ${ }^{\circledR}$ were commonly used to treat pain. Reassurance, positioning and immobilization were used as alternatives to drugs. Prehospital pain management could be improved by addressing practitioner and patient barriers, increasing available drugs and developing multiorganizational pain management protocols supported by training for staff.

\section{Conclusions}

Pain is often poorly managed and undertreated in the prehospital environment. Our findings may be used to inform guidance, education and policy to improve the prehospital pain management pathway. 


\section{INTRODUCTION}

Eighty percent of patients have been found to experience pain during ambulance transport;[1] and 20 percent report moderate to severe pain.[2] Twenty percent of those surveyed by a UK regulatory body stated that they would have liked more pain relief and $5 \%$ felt that ambulance staff could have done more to control their pain.[1]

National prehospital guidelines developed by the Joint Royal Colleges Ambulance Liaison Committee [JRCALC] emphasise the importance of early treatment of pain.[3] Early treatment has been shown to reduce pain in the emergency department [ED] and is a quality indicator.[4] Pain management is a priority for research[5] and quality improvement[6] in urgent settings but is often inadequate[7]: in a recent study, pain was present in 70 percent of patients presenting to an ambulance service, but was treated with analgesics in under 40 percent.[8] Another study showed that significant pain relief was achieved in just 40 percent of patients.[9]

Management of pain includes assessment using rating scales[10] and treatment with non-drug measures and analgesics. Barriers to better pre-hospital management of pain include practitioners' beliefs about pain severity[11] leading to underestimation of pain,[12] patient factors such as low initial pain score[13] or impaired consciousness,[10] organisational factors such as prolonged on-scene times with intravenous analgesia,[14] and systematic barriers to implementing guidelines.[13]

There have been few qualitative studies of pain management in prehospital or emergency settings in the US, UK or elsewhere[11,15,16] and no previous studies have explored the pathway of prehospital pain management from home to the hospital ED with patients, ambulance staff and emergency department staff. The objective of this study was to investigate patient and practitioner views on their experiences of pain management including facilitators and barriers in order to improve care and develop a more patient centred approach to treatment.

This study aimed to explore the following questions (a) What are the attitudes, beliefs, expectations and concerns of patients and emergency healthcare staff in relation to prehospital management of pain? (b) What constitutes optimal pain management in the prehospital setting from patient, practitioner and system perspectives? (c) What are the barriers and facilitators to optimal pain management in the pre-hospital setting from patient, practitioner and system perspectives?

\section{METHOD}

\section{Design}

We used a qualitative design adopting a constructivist view and an interpretive phenomenological approach.[17] We were interested in the lived experience of prehospital care for pain, what happened during such healthcare episodes and the meaning for those involved, focusing on how this experience might be improved.[18] We explored and compared the breadth and depth of experiences and beliefs relating to prehospital assessment and treatment of pain as well as participants' perceptions of facilitators and/or barriers associated with the management of pain. We used separate semistructured focus groups or interviews for patients, ambulance and ED clinicians; one-to-one individual semi-structured interviews were used to further explore the issues identified in the focus groups since the latter can be dominated by some participants or perceived group norms. 


\section{Participants}

The study took place during 2008/09 in the East Midlands, UK and involved one regional ambulance service using JRCALC guidelines[3] and one emergency department, i.e. a single system/single site study. We used purposive sampling aiming to maximise participant variation (age, gender, professional background of clinician or health status of patient). We retrieved clinical records for adult patients transported to hospital by ambulance during the previous six months with pain due to a suspected fracture or cardiac chest pain. Patients were contacted by letter after agreement from their GP to ensure that patients were fit to be interviewed. Ambulance clinicians (paramedics and emergency technicians of varying experience and seniority) were chosen at random using identification numbers from the staff database. Emergency department clinicians included doctors and nurses of varying experience and seniority were selected from the local hospital through contact with the consultant. Participants were invited by letter to attend an interview and interviews were continued until data saturation was achieved.

\section{Data collection and analysis}

Interviews were conducted by two non-practising clinicians (AS, MI) using a previously piloted topic guide. Clinician groups included 5-8 participants and patient groups, 3-4 participants. Individual and group interviews with the three categories of participant took place in parallel to enable constant comparisons within and between groups. Interviews were tape recorded and transcribed verbatim; relevant field notes were taken.

Transcripts were read and re-read highlighting statements that related to the phenomenon of pain. These were coded and analysed using thematic content analysis supported by computer qualitative data analysis software, MAXQDA.[19] Cross checking of transcripts with recorded interview audio files and field notes was carried out during coding.

Analysis was undertaken by three researchers independently while data collection was in progress. A coding framework was developed inductively from the data.[20] Initial codes (and their associated memos or descriptions) were used to develop a coding book. Further transcripts were analysed (by $\mathrm{MI}$ ) and new codes developed iteratively using the constant comparative method. Participants continued to be recruited until no new codes emerged. Codes were arranged and organised hierarchically to form themes. The final categories were agreed through iterative discussion.

We wanted to identify the areas of agreement between and within patient and clinician groups as well as seeking and identifying 'deviant cases' where there was disagreement between participants.[18] We did not undertake respondent validation because we felt that this would not add to the analysis.

\section{RESULTS}

Fifty five participants were interviewed. These included 17 patients (men, women: 10, 7 ) and 38 practitioners comprising 25 ambulance clinicians (14 paramedics, 11 emergency technicians) and 13 ED clinicians (9 physicians, 4 nurses).

In all, five focus groups were conducted with patients (2 groups), ambulance clinicians (2 groups) and ED clinicians (1 group). Twenty eight semi-structured one-to-one interviews were conducted with 10 patients, 12 ambulance clinicians and 6 ED clinicians [Appendix 1]. 
Patients varied in age (35 to 76 years), gender and health status: there were 13 patients with AMI and 4 patients with fracture. Ambulance staff included paramedics, technicians, community practitioners, emergency care practitioners, managers and clinical education specialists; ED staff included doctors and nurses of varying experience [Appendix 2].

Themes are described under five main categories: consider expectations and beliefs of patients and staff in pain management; widen pain assessment strategies; optimise non-drug treatment options for pain; implement and increase options for drug treatment for pain; and enhance communication and coordination along the prehospital pain management pathway.

\section{Consider expectations and beliefs of patients and staff in pain management}

Patients expected and clinicians perceived an expectation of immediate pain relief in the ambulance before transport to hospital.

I was just grateful to have some relief from that pain... it was something I couldn't deal with...um....wanted relief. Patient Interview 10

They [patients] have a greater expectation of pain relief now ....... think ambulances will need to be actively involved in pain relief. Paramedic Interview 2

Reassurance and a professional approach helped calm patients and alleviate pain. A preference to be treated at home or avoid hospital often influenced management options.

I'd been trying unsuccessfully to relieve my own pain... I looked to them [paramedics] to deal with it, to relieve my anxieties, to reassure me. Patient Interview 10

Some people ...will ring an ambulance for some pain relief ..."I don't want to go to hospital" ..."can't you give me something"... we [Paramedics] can give you some morphine but we have to take you [to hospital]. "Oh no, no I don't want to go to hospital. Can you give me a tablet?"[at home] Paramedic Interview 6

Early pain relief facilitated subsequent handling or transfer in the ED, better management and contributing to improved outcomes. It was important that good pain relief was equitable, including for those who did not complain in spite of being in pain.

[The] patient can explain things ... better if they're not in pain ...l can make a better assessment .... If it's not managed then I've got to deal with pain relief first before I can do anything else. ED Clinician Interview 3

It's ethical that every patient should get analgesia at a fairly early stage and ...very often [some patients] don't complain about pain. Just because they're not complaining ... doesn't mean ... they haven't got pain... Paramedic Interview 11

Conflicting expectations and beliefs sometimes hampered prehospital pain care. Patients lacked awareness of available options; some were unsure about what constituted pain relief and thought analgesics had been denied even though they had been administered 'gas and air' (Entonox ${ }^{\circledR}$ : nitrous oxide/oxygen mixture). Some patients wished to defer pain relief until definitive treatment thinking that early analgesia 
might affect subsequent treatment or prevent administration of more effective pain relief at hospital.

They didn't give me pain relief...they gave me gas and air ... but I didn't get pain killers...until I got to hospital. Patient interview 6

A lot of them [patient] seem to be convinced that it'll affect what they can do in hospital ... it might stop ... having something else ... in hospital. Paramedic Focus Group 1

Patients sometimes accepted inadequate analgesia, believing this was due to limited time, resources or scope of ambulance practitioners' practice. Pain management skills of clinicians varied and sometimes other priorities conflicted with pain management, for example with time critical and life threatening conditions ED clinicians perceived that severe pain was treated better by ambulance staff compared to mild and moderate pain.

Well I think...many circumstances are beyond their [paramedics] control because of the limited time and resources... Their job isn't to make the condition better... their job is to get the patient... where they could make that person better... Patient Interview 5

Sometimes it can be time critical. .. there are injuries or potentially life threatening [condition] that you need to be off without giving that pain relief....and that is an assessment you have to make... a bit of pain initially could save their life. Paramedic Interview 6

Generally they've well managed the major problem .... but minor and moderate pain ... are not really sort of treated adequately ...there are different varieties in ambulance crews you see...some are really good, some are not! ED Clinician Focus Group

"Gas and air" (Entonox ${ }^{\circledR}$ ) was often expected for milder pain whereas tablets or injections were anticipated if these had been received previously. Media depictions raised patients' expectations that injection/intravenous drugs would be administered.

It was not a lot of pain and they [patients] expected gas and air. Paramedic2

I expected them to give me a drug [tablet] to try and ease the pain. Whether they were permitted to do so I don't know. Patient Interview 4

\section{Widen pain assessment strategies}

Some patients found verbal pain scores, zero (0) signifying no pain and 10 as the worst pain imaginable, easy and others were confused. Paramedics did not rely solely on the pain score but also took other observations into account: body language, facial expression and pain on movement were compared with clinical signs including pulse, blood pressure and skin colour as part of the assessment.

The ambulance man ... he sort of asked me 'is it [pain] a two or is it a three' and I was thinking 'is this out of what?'... Patient interview 1

I do tend to look at more ... their [patients'] whole body language and facial expressions Paramedic Interview 2 
Causation of pain or apparent severity of injury often helped determine whether or what pain relief should be given.

Obviously how bad it [injury] looks ...you can see it's [bone] broken in half... thinking that must be hurting a lot ...Paramedic Interview 8

Variation in pain threshold and the perception that patients reportedly exaggerated or underplayed pain created a dissonance between pain score and non-verbal or clinical features.

You get the elderly lady with a fractured hip ... but goes 'it's just a bit of a twinge' and yet you'll get the body builder who's broken his finger who's screaming... difficult isn't it? Paramedic Interview 5

Lack of cooperation or a language barrier sometimes hindered assessment. Older people or those with chronic condition(s), e.g. arthritis, were felt to underestimate their pain. Conditions such as learning disability and dementia made pain assessment more difficult and emotional or social factors and alcohol or non-prescription drugs (of abuse) could affect patients' perception of pain.

[The] older generation underplay their pain... they're used to living with pain... Paramedic Interview 5

"[Paramedic 5]: I think...the language barrier is such a hindrance to us in every aspect" Paramedic Focus Group 1

I think if they've been drinking... alcohol...if they've ...got pain. It's an anaesthetic in itself so it's difficult to assess the condition. Paramedic Interview 10

\section{Optimise non-drug treatment options for pain}

Non-drug management played an important role in pre-hospital pain management. Reassurance, information (normal test results) or distraction sometimes helped. Physical contact by putting a hand on a shoulder or holding a hand, making eye contact or talking increased confidence and provided comfort.

They reassured me that I wasn't wasting anybody's time...they kept me informed ... what was going on and what they were going to do next and when I was taken into hospital ... continuous reassurance and information ... helped. Patient interview 5

[Paramedic 3:] "Distraction is a very good way of diverting them [patient] away from pain. Talk about something completely different and they forget all about it.

Paramedic Focus Group 1

Positioning, supporting or immobilising the patient was important, especially in the case of fracture. Patients own coping strategies, through personal knowledge or past experiences, often helped to reduce pain.

I hurt my ankle ....they splinted...it was immobile. ... it did feel very supportive...it made my leg feel comfortable. It got a bit heavy after a while... but it was quite comfy. Patient Interview 1 


\section{Implement and increase options for drug treatment for pain}

Availability of analgesics depended on type and training of ambulance personnel and ambulance guidelines or Patient Group Directions (PGD). Inhaled Entonox ${ }^{\circledR}$, intravenous morphine, and oral drugs including oral morphine and acetaminophen [paracetamol] (for children) were commonly used. Paramedics found Entonox easy to use and rapidy effective. As its effects were short term ED clinicians replaced it with a longer acting drug on arrival at hospital but endorsed its use during transportation. Clinicians and patients considered morphine to be the most effective analgesic and anxiolytic; it was used pre-emptively to reduce pain with movement during the journey or patient handling.

They [paramedics] used to use Entonox ${ }^{\circledR} \ldots$ but ...we do tend to take it straight off them as soon as they [patient] come in [A\&E] and give them something more long term ... but then for the crew it suits them it's a short term thing. ED Clinician Interview 3

Query fractured hip... so I do give morphine, ... for a fact that afterwards they've got to be manipulated ... in hospital...then you move them then they've got pain. I like to pre-empt that. Paramedic 5, Focus Group 2

Paramedics often selected drugs according to the cause, rather than severity of pain: Entonox ${ }^{\circledR}$ was given for fractures whereas intravenous morphine was used for cardiac pain. Ambulance clinicians also selected drugs according to anticipated travel time, route and distance to hospital; they preferred morphine for greater travel times or rougher roads whereas Entonox ${ }^{\circledR}$ was selected for shorter distances.

If it's chest pain we go straight into morphine or Oramorph. If it's a broken leg or limb or whatever then it's straight on to the Entonox ${ }^{\circledR}$ first depending on the patient's condition. Paramedic Interview 9

Often patients self medicated before the ambulance arrived and failure of self-treatment was what led to a call for help. Non-analgesics such as glyceryl trinitrate (GTN) also helped to relieve pain.

I do have angina and I had my angina spray but it didn't do any good ...I could feel the colour draining out of my face and feeling woozy so he [patient's spouse] ... rang an ambulance. Patient interview 6

Barriers to drug treatment included patient refusal, concern about adverse effects, contraindications or interactions, false beliefs (e.g. addiction with morphine) and previous clinical experience of severe reactions to morphine. ED clinicians were concerned about paramedics using Entonox ${ }^{\circledR}$ where it was contraindicated, e.g. for bowel obstruction.

[Paramedic 4]: “I think they're [patients] confused about what morphine is... it's like you've offered them crack cocaine or something. 'No, no I [patient] can't have that'. Paramedic Focus Group 1

Your crews give Entonox ${ }^{\circledR}$ willy-nilly. Like for abdominal pains and it's contraindicated within the trust...we don't give Entonox to abdominal pain and yet you do. ED Clinician Interview 1

Some paramedics expressed frustration at the limited choice of drugs; technicians had 
to use basic pain relief options or wait for a qualified crew or doctor to arrive.

[Paramedic 1]: Then if you're a CP [Community Practitioner] or an ECP [Emergency care Practitioner] you've got paracetamol, dicloflenac...should that be a wide range of drugs that we carry as a paramedic? Paramedic Focus Group 1

if you've got somebody who can't give morphine ...like a double tech[nician] crew ...they can't give it, they can't cannulate. That could be a problem. ED Clinician Interview 3

Some patients were deemed difficult to treat with intravenous morphine because of hypotension (elderly), calculating dosage (children, obese or underweight) and potential abuse (suspected addicts).

I think our paediatric pain relief is a problem because ... morphine is not available for children. Paramedic Interview 2

\section{Enhance communication and coordination along the pain management pathway}

A number of ideas emerged for improving the pain pathway. Good communication of information from ambulance staff was valued by ED clinicians; what, if any, treatment had been offered or given and what effect had this had. This helped subsequent ED management, particularly when patients were not in a condition to report this information.

I got to hospital and was assessed. The team when I got to hospital knew the details of why I was brought in .... and my condition was stabilised so ... they've [paramedic] done their job and you can't ask any more than that. Patient interview 5

When they [patient] come through those doors on an ambulance trolley you can see what position they're in, whether things have been splinted or supported. And the crews will tell us that 'we've had to do this' to the patient. It has relieved the pain to some extent but we were unable to get any further pain relief." $E D$ Clinician Interview 4

Patients appreciated concern shown, structured questions and early assessment and intervention for pain. Maintaining dignity was also important although personal privacy was sometimes difficult to maintain during treatment.

So all I was aware of was that l'd got an audience... four children looking....and seeing what was going on. I just thought we ought to have had a bit of privacy but I was in too much pain you get on with what you're doing. Patient interview 2

Clinical observation sometimes conflicted with patients' reported pain and some felt that practitioners' clinical judgment could play a greater role in pain assessment.

[Paramedic 7]: I think there should be some sort of comparison. Patient's opinion on what their pain is and what the clinician's opinion on what pain is. What they think it should be. Paramedic Focus Group 1

Paramedics felt that PGDs enabling wider use of analgesics, muscle relaxants and antispasmodics would enable more patients to be treated including at home. Newer 
drugs and alternative administration routes could be helpful for patients who refused injections or special groups such as elderly or children.

A paramedic can give Entonox ${ }^{\circledR}$ or Oramorph or morphine but a [emergency care] practitioner can give paracetamol, codeine, dicloflenac, morphine, Entonox, Oramorph, co-codamol. I'd like to see some of them brought in for all paramedics to use. There's minimal training needed and they're all capable of it...so that would be useful. Paramedic Interview 4

Many felt that shared protocols and training involving both ambulance and ED would help reduce inappropriate prehospital cannulation which hindered further venous access. ED staff sometimes felt that morphine was not indicated or should have been delayed until the ED.

One thing that for us they [ambulance service] should be thinking about is to ... get it line with our protocols and work to a joint protocol ....if they [patient] have clinical indications... may be they should do [treat] that from the start ... maybe could just tie in our protocol and that would be quite good...we could have the same protocol. ED Clinician Focus Group

\section{DISCUSSION}

\section{Main findings}

This is the first study to undertake a comparative investigation of patients' and practitioners' views of prehospital pain management. Improvement in urgent pain management will require services to consider the expectations and beliefs of patients and staff in pain management, widen pain assessment strategies, optimise non-drug treatment options for pain, implement and increase options for drug treatment for pain and enhance communication and coordination along the prehospital pain management pathway.

Many patients and healthcare staff expected pain to be relieved in the ambulance but refusal of or acceptance of inadequate analgesia also happened because patients were concerned about adverse drug effects, did not want to be transported when this was required after drug [morphine] administration, or were worried that pain relief would interfere with subsequent hospital assessment. Some patients and practitioners found pain scores confusing and when clinical observations were discordant with reported pain scores, practitioners often responded according to presumed diagnosis rather than pain severity leading to over or under treatment.

Barriers to assessment included communication difficulties, non-cooperation or the influence of alcohol or drugs. Morphine and Entonox ${ }^{\circledR}$ were commonly used to treat pain but reassurance, positioning and immobilization were often used as alternatives to drugs. Suggestions to improve prehospital pain management included addressing identified barriers, increasing drug options and developing agreed multi-organizational pain management protocols with appropriate training for staff.

\section{Comparison with existing literature}

Pain is a subjective phenomenon and intensity is modified by a number of social, psychological and environmental factors. Other studies confirm that patients want and practitioners understand the importance of early and effective pain relief in 
emergencies[21,22] not least because this has been shown to affect outcomes at discharge.[23] Prehospital triage systems often do not prioritise pain[24] and prehospital analgesia although it enhances pain relief,[25,26] is underutilised.[8,27,28] Pain assessment and analgesia have been shown to be worse for older adults,[29] women,[30] ethnic minorities[31] and in some[32] but not all[33] studies of children and these findings are partly explained by our findings of communication and language difficulties as well as the complexity of pain assessment and treatment.

Practitioners in our study took into account this complexity by acknowledging a number of issues over and above reported pain scores when deciding on analgesia such as initial severity,[13,34] time to hospital, chronicity or underlying cause as in other studies.[11] Prehospital assessment of pain is feasible[10] and increases the likelihood of analgesia.[35] Jones and colleagues in a small study of six interviewees found, as we did, that paramedics used a variety of assessment strategies such as non-verbal and physiological signs in addition to a pain score.[11] We found that practitioners also used these means to refine their assessment although previous studies suggest that practitioner assessment and vital signs are unreliable for assessing pain.[36] Practitioner assessments tend to underestimate analgesic requirements[37,38] and under-assessment is associated with oligoanalgesia.[39] Evidence is also lacking for appropriate provision of analgesia in certain clinical conditions such as head injury where conscious level and communication may be impaired.[13]

Entonox ${ }^{\circledR}$ and opiates are widely used prehospitally.[40] Both are safe[41,42] but need to be used with caution or are contraindicated in some conditions and variation in individual response can lead to undertreatment.[34] Patients' concerns about possible effects of analgesia on subsequent diagnosis is unfounded.[43] Provision of analgesia is known to increase on-scene times[44] and over-medication for trauma can have deleterious effects.[45] Participants suggested widening available options for analgesia and although new drugs and routes of administration[46-48] are endorsed by some experts,[49] others have emphasized the importance of education and training[34] to better implement the range of drugs currently available.[50]

Practitioners in our study also referred to non-drug measures which have also been shown to assist management in previous studies, including psychological techniques,[11] splints[40] and positioning[51].

\section{Strengths and limitations}

The study included patients and clinicians who had recently experienced emergency treatment for pain. The purposive sampling strategy used and triangulation of views of a range of clinicians and service users enable wider conclusions to be drawn from the data.[52] Participants' contributions may have been affected by recall bias but their recent experiences were nevertheless relevant. This was a single system/single region study involving one regional ambulance service and a single emergency department so views may not reflect those of staff in other areas. The two interviewers [male and female] were not practising clinicians; one was a researcher [non-practicing medical doctor] and the other a manager [physiotherapist]. Both were careful not to influence responses but their professional background may have affected some of the participants' contributions. 


\section{Implications for future practice and research}

Our findings may inform guidelines, education and policy for improvement.[6,53] Educational interventions have successfully shown improvements in knowledge and performance of ED[54] and prehospital pain management[55] and systematic initiatives have been successfully implemented, even on a national scale.[56]

Our findings suggest that verbal pain scores may be unreliable when used in isolation and that the range of analgesics used prehospitally should be reviewed. Further research needs to be conducted to develop better assessment tools, patient reported outcome measures and pathways for prehospital pain management using a range of methods appropriate to each.

\section{Conclusions}

Pain is often poorly managed and undertreated in the prehospital environment. Pain scores are sometimes poorly administered by clinicians and ill understood by patients; they overlook important information for deciding appropriate treatment such as causation of pain or non-verbal features suggesting more severe pain. Better management of pain could be achieved by incorporating a wider ranger of information from both patients and clinicians, providing better information for patients on treatment options and by considering the whole pathway of care from home to hospital. Our findings may be used to inform guidance, education and policy to improve the prehospital pain management pathway. 


\section{Licence for publication}

The Corresponding Author has the right to grant on behalf of all authors and does grant on behalf of all authors, an exclusive licence (or non exclusive for government employees) on a worldwide basis to the BMJ Publishing Group Ltd and its Licensees to permit this article (if accepted) to be published in BMJ Quality and Safety and any other BMJPGL products to exploit all subsidiary rights, as set out in our licence (http://emj.bmjjournals.com/ifora/licence.dtl).

\section{Authors' contributions}

ANS conceived the original idea for the study. AS and MI undertook the interviews and focus groups. All authors contributed to the design and conception of the paper. ANS wrote the first draft of the paper. All authors contributed to the discussion and final paper. ANS is guarantor for the study.

\section{Ethical approval}

This study was approved by Nottingham Research Ethics Committee 2 (07/H0408/112) and the Ethics Committee of the University of Lincoln. Approval for Research Management and Governance was sought and gained from NHS Lincolnshire, East Midlands Ambulance Services NHS Trust and United Lincolnshire Hospitals NHS Trust.

\section{Competing interests}

None.

\section{Funding}

The study was funded by Trent Research and Development Support Unit and East Midlands Ambulance Service NHS Trust. 
References

1. Healthcare Commission. Patient survey report 2004 - ambulance services 2004. Available at: http://www.cqc.org.uk/. Accessed April 7, 2005.

2. McLean SA, Maio RF, Domeier RM. The epidemiology of pain in the prehospital setting. Prehosp Emerg Care 2002;6:402-5.

3. Joint Royal Colleges Ambulance Liaison Committee, Ambulance Service Association. UK Ambulance Service Clinical Practice Guidelines. London: Ambulance Service Association, 2006.

4. Tcherny-Lessenot S, Karwowski-Soulie F, Lamarche-Vadel A et al. Management and relief of pain in an emergency department from the adult patients' perspective. J Pain Symptom Manage 2003;25:539-46.

5. Snooks H, Evans A, Wells B et al. What are the highest priorities for research in pre-hospital care? Results of a review and Delphi consultation exercise (accessed 13 January 2009). Journal of Emergency Primary Care (JEPHC) 2008;6:1-20.

6. McManus JG, Jr., Sallee DR, Jr. Pain management in the prehospital environment. Emerg Med Clin North Am 2005;23:415-31.

7. Rupp T, Delaney KA. Inadequate analgesia in emergency medicine. Ann Emerg Med 2004;43:494-503.

8. Siriwardena AN, Shaw D, Bouliotis G. Exploratory cross-sectional study of factors associated with pre-hospital management of pain. J Eval Clin Pract 2010.

9. Jennings PA, Cameron P, Bernard S. Epidemiology of prehospital pain: an opportunity for improvement. Emerg Med J 2011;28:530-1.

10. McLean SA, Domeier RM, DeVore HK et al. The feasibility of pain assessment in the prehospital setting. Prehosp Emerg Care 2004;8:155-61.

11. Jones GE, Machen I. Pre-hospital pain management: the paramedics' perspective. Accid Emerg Nurs 2003;11:166-72.

12. Puntillo K, Neighbor M, O'Neil N et al. Accuracy of emergency nurses in assessment of patients' pain. Pain Manag Nurs 2003;4:171-5.

13. Frakes MA, Lord WR, Kociszewski $C$ et al. Factors associated with unoffered trauma analgesia in critical care transport. Am J Emerg Med 2009;27:49-54.

14. Chambers JA, Guly HR. Prehospital intravenous nalbuphine administered by paramedics. Resuscitation 1994;27:153-8.

15. Graham J. Adult patients' perceptions of pain management at triage: a small exploratory study. Accid Emerg Nurs 2002;10:78-86. 
16. Wilsey $\mathrm{BL}$, Fishman $\mathrm{SM}$, Crandall $\mathrm{M}$ et al. A qualitative study of the barriers to chronic pain management in the ED. Am J Emerg Med 2008;26:255-63.

17. Reeves S, Albert M, Kuper A et al. Why use theories in qualitative research? BMJ 2008;337:a949.

18. Mays N, Pope C. Qualitative research in health care. Assessing quality in qualitative research. BMJ 2000;320:50-2.

19. MAXQDA 2007. Udo Kuckartz,Berlin [Available from: URL:<http://www.maxqda.com>

20. Glaser BG, Strauss AL. The discovery of grounded theory: strategies for qualitative research. Chicago: Aldine De Gruyter, 1967.

21. Fosnocht DE, Heaps ND, Swanson ER. Patient expectations for pain relief in the ED. Am J Emerg Med 2004;22:286-8.

22. Beel TL, Mitchiner JC, Frederiksen SM et al. Patient preferences regarding pain medication in the ED. Am J Emerg Med 2000;18:376-80.

23. Hall-Lord ML, Larsson BW, Baath $\mathrm{C}$ et al. Experiences of pain and distress in hip fracture patients. J Orth Nurs 2004;8:221-30.

24. Lord B, Cui J, Woollard M. Ambulance call triage outcomes for patients reporting pain: a retrospective cross-sectional analysis of pain score versus triage level. Emerg Med J 2009;26:123-7.

25. Abbuhl FB, Reed DB. Time to analgesia for patients with painful extremity injuries transported to the emergency department by ambulance. Prehosp Emerg Care 2003;7:445-7.

26. Fullerton-Gleason L, Crandall C, Sklar DP. Prehospital administration of morphine for isolated extremity injuries: a change in protocol reduces time to medication. Prehosp Emerg Care 2002;6:411-6.

27. White LJ, Cooper JD, Chambers RM et al. Prehospital use of analgesia for suspected extremity fractures. Prehosp Emerg Care 2000;4:205-8.

28. Vassiliadis J, Hitos K, Hill CT. Factors influencing prehospital and emergency department analgesia administration to patients with femoral neck fractures. Emerg Med (Fremantle ) 2002;14:261-6.

29. Jones JS, Johnson $\mathrm{K}$, McNinch $\mathrm{M}$. Age as a risk factor for inadequate emergency department analgesia. Am J Emerg Med 1996;14:157-60.

30. Michael GE, Sporer KA, Youngblood GM. Women are less likely than men to receive prehospital analgesia for isolated extremity injuries. Am J Emerg Med 2007;25:901-6.

31. Green CR, Anderson KO, Baker TA et al. The unequal burden of pain: confronting racial and ethnic disparities in pain. Pain Med 2003;4:277-94. 
32. Izsak E, Moore JL, Stringfellow $\mathrm{K}$ et al. Prehospital pain assessment in pediatric trauma. Prehosp Emerg Care 2008;12:182-6.

33. Swor R, McEachin CM, Seguin D et al. Prehospital pain management in children suffering traumatic injury. Prehosp Emerg Care 2005;9:40-3.

34. Bounes $\mathrm{V}$, Barniol $\mathrm{C}$, Minville $\mathrm{V}$ et al. Predictors of pain relief and adverse events in patients receiving opioids in a prehospital setting. Am J Emerg Med 2010.

35. Silka PA, Roth MM, Moreno G et al. Pain scores improve analgesic administration patterns for trauma patients in the emergency department. Acad Emerg Med 2004;11:264-70.

36. Lord $\mathrm{B}$, Woollard $\mathrm{M}$. The reliability of vital signs in estimating pain severity among adult patients treated by paramedics. Emerg Med J 2011;28:147-50.

37. Luger TJ, Lederer W, Gassner M et al. Acute pain is underassessed in out-ofhospital emergencies. Acad Emerg Med 2003;10:627-32.

38. Bijur PE, Berard A, Esses D et al. Lack of influence of patient self-report of pain intensity on administration of opioids for suspected long-bone fractures. J Pain 2006;7:438-44.

39. Hennes H, Kim MK, Pirrallo RG. Prehospital pain management: a comparison of providers' perceptions and practices. Prehosp Emerg Care 2005;9:32-9.

40. Roberts K, Allison KP, Porter KM. A review of emergency equipment carried and procedures performed by UK front line paramedics. Resuscitation 2003;58:1538.

41. Faddy SC, Garlick SR. A systematic review of the safety of analgesia with $50 \%$ nitrous oxide: can lay responders use analgesic gases in the prehospital setting? Emerg Med J 2005;22:901-8.

42. Bounes V, Charpentier S, Houze-Cerfon $\mathrm{CH}$ et al. Is there an ideal morphine dose for prehospital treatment of severe acute pain? A randomized, double-blind comparison of 2 doses. Am J Emerg Med 2008;26:148-54.

43. Thomas SH, Silen W, Cheema F et al. Effects of morphine analgesia on diagnostic accuracy in Emergency Department patients with abdominal pain: a prospective, randomized trial. J Am Coll Surg 2003;196:18-31.

44. Johnson GS, Guly HR. The effect of pre-hospital administration of intravenous nalbuphine on on-scene times. J Accid Emerg Med 1995;12:20-2.

45. Lucas CE, Vlahos AL, Ledgerwood AM. Kindness kills: the negative impact of pain as the fifth vital sign. J Am Coll Surg 2007;205:101-7.

46. Borland ML, Jacobs I, Geelhoed G. Intranasal fentanyl reduces acute pain in children in the emergency department: a safety and efficacy study. Emerg Med (Fremantle ) 2002;14:275-80. 
47. Woollard $\mathrm{M}$, Jones $\mathrm{T}$, Pitt $\mathrm{K}$ et al. Hitting them where it hurts? Low dose nalbuphine therapy. Emerg Med J 2002;19:565-70.

48. Vergnion $M$, Degesves $S$, Garcet $L$ et al. Tramadol, an alternative to morphine for treating posttraumatic pain in the prehospital situation. Anesth Analg 2001;92:1543-6.

49. Borland ML, Jacobs I, Rogers IR. Options in prehospital analgesia. Emerg Med (Fremantle ) 2002;14:77-84.

50. Bledsoe B, Braude D, Dailey MW et al. Simplifying prehospital analgesia. Why certain medications should or should not be used for pain management in the field. JEMS 2005;30:56-63.

51. Lerner EB, Billittier AJ, Moscati RM. The effects of neutral positioning with and without padding on spinal immobilization of healthy subjects. Prehosp Emerg Care 1998;2:112-6.

52. Kuper A, Lingard L, Levinson W. Critically appraising qualitative research. BMJ 2008;337:a1035.

53. Ricard-Hibon A, Chollet C, Saada S et al. A quality control program for acute pain management in out-of-hospital critical care medicine. Ann Emerg Med 1999;34:738-44.

54. Kelly AM. A process approach to improving pain management in the emergency department: development and evaluation. J Accid Emerg Med 2000;17:185-7.

55. French SC, Salama NP, Baqai S et al. Effects of an educational intervention on prehospital pain management. Prehosp Emerg Care 2006;10:71-6.

56. Ricard-Hibon A, Ducasse JL, Ravaud P et al. Quality control programme for acute pain management in emergency medicine: a national survey. Eur J Emerg Med 2004;11:198-203. 